\title{
INTEGRAL observations of five sources in the Galactic Center region
}

\author{
A. Lutovinov ${ }^{1}$, M. Revnivtsev ${ }^{1,2}$, S. Molkov ${ }^{1}$, and R. Sunyaev ${ }^{1,2}$ \\ 1 Space Research Institute, Russian Academy of Sciences, Profsoyuznaya 84/32, 117810 Moscow, Russia \\ e-mail: lutovinov@hea.iki.rssi.ru \\ 2 Max-Planck-Institute für Astrophysik, Karl-Schwarzschild-Str. 1, 85740 Garching bei München, Germany
}

Received 16 July 2004 / Accepted 10 September 2004

\begin{abstract}
A number of new X-ray sources (IGR J17091-3624, IGR/XTE J17391-3021, IGR J17464-3213 (=XTE J17464-3213 $=$ H 1743-322), IGR J17597-2201, SAX/IGR J18027-2017) have been observed with the INTEGRAL observatory during ultra deep exposure of the Galactic Center region in August-September 2003. Most of them were permanently visible by INTEGRAL at energies higher than $\sim 20 \mathrm{keV}$, but IGR/XTE J17391-3021 was observed only during its flaring activity with a flux maximum of $\sim 120$ mCrab. IGR J17091-3624, IGR J17464-3213 and IGR J17597-2201 were detected up to 100-150 keV. In this paper we present the analysis of INTEGRAL observations of these sources to determine the nature of these objects. We conclude that all of them have a galactic origin. Two sources are black hole candidates (IGR J17091-3624 and IGR J17464-3213), one (IGR J17597-2201) is an LMXB neutron star binary (presumably an X-ray burster) and two other sources (IGR J17391-3021 and SAX/IGR J18027-2017) are neutron stars in high mass binaries; one of them (SAX/IGR J18027-2017) is an accreting $\mathrm{X}$-ray pulsar.
\end{abstract}

Key words. Galaxy: center - stars: binaries: general - X-rays: binaries

\section{Introduction}

About 12 new hard X-ray sources have been discovered with the INTEGRAL observatory. Most of them are concentrated towards the Galactic plane, Galactic Center and spiral arm tangents (see Revnivtsev et al. 2004a; Molkov et al. 2004; Tomsick et al. 2004; Bird et al. 2004, and references therein). Such a concentration strongly suggests that the majority of these sources have a galactic origin.

An ultra deep survey of the Galactic Center region, performed by the INTEGRAL in 2003 provided very important information about the population of Galactic X-ray sources in the bulge and revealed a number of transients in this field. A previous deep survey of this region in hard X-ray/ soft gamma-rays was made by the SIGMA telescope aboard the GRANAT orbital observatory with a sensitivity of 3$5 \mathrm{mCrab}$ in the $40-150 \mathrm{keV}$ energy band and allowed us to detect 20 sources including transients (Churazov et al. 1994; Revnivtsev et al. 2004b). Recent analysis of the images of the Galactic Center region obtained by INTEGRAL/IBIS in the energy range $18-60 \mathrm{keV}$ with a typical sensitivity of 1-2 mCrab revealed 60 sources of different natures: low-mass X-ray binaries, X-ray pulsars, black-hole candidates, cataclysmic variables, extragalactic sources etc. (Revnivtsev et al. 2004a). The nature of a significant part of the detected sources is still unclear. Out of 60 detected sources six new sources were detected during INTEGRAL observations of the Galactic Center region. Five of them were statistically significantly detected on the averaged map of the region, while IGR J17544-2619 demonstrated a very peculiar behavior (Sunyaev et al. 2003a; Grebenev et al. 2003a; in't Zand et al. 2004; Gonzalez-Riestra et al. 2004). It was detected only during a few ks of observations and we do not discuss it here.

In this paper we summarize the information about properties of new INTEGRAL sources and try to determine the nature of these sources.

\section{Observations and data reduction}

The international gamma-ray observatory INTEGRAL (Winkler et al. 2003) was launched to the orbit with the Russian rocket PROTON from the cosmodrom Baikonur on Oct. 17, 2002 (Eismont et al. 2003). It consists of four instruments designed for investigation of galactic and extragalactic sources in a wide $\mathrm{X}$-ray $/ \gamma$-ray energy band from 3 to $10000 \mathrm{keV}$ : the gamma-telescope IBIS, the SPI spectrometer, the X-ray monitor JEM-X and the optical monitor OMC. In this paper we used data from the ISGRI detector (Lebrun et al. 2003) of the IBIS telescope (Ubertini et al. 2003). Most sources were outside the JEM-X field of view or were too faint for the detection with this instrument. 
Table 1. Journal of observations.

\begin{tabular}{l|l|l|c|c|c|c|l}
\hline \hline Source & $\mathrm{RA}^{a}$ & Dec $^{a}$ & \multicolumn{2}{|c|}{2003} & \multicolumn{2}{|c|}{2004} & \multirow{2}{*}{ Source type } \\
\cline { 4 - 7 } & $(2000)$ & $(2000)$ & Exp., ks $^{b}$ & Flux, mCrab & Exp., ks & Flux, mCrab & \\
\hline IGR J17091-3624 & $17^{\mathrm{h}} 09^{\mathrm{m}} 06^{\mathrm{s}}$ & $36^{\circ} 24^{\prime} 38^{\prime \prime}$ & 920 & $7.6 \pm 0.2$ & 96 & $16.8 \pm 0.6$ & BHC \\
IGR/XTE J17391-3021 & $17^{\mathrm{h}} 39^{\mathrm{m}} 06^{\mathrm{s}}$ & $30^{\circ} 21^{\prime} 30^{\prime \prime}$ & 2055 & $1.6 \pm 0.1(123 \pm 3)^{d}$ & 236 & $1.1^{e}$ & NS, HMXB? \\
IGR J17464-3213 & $17^{\mathrm{h}} 46^{\mathrm{m}} 24^{\mathrm{s}}$ & $32^{\circ} 13^{\prime}$ & 1979 & $6.0 \pm 0.1(43 \pm 5)^{d}$ & 219 & $0.5^{e}$ & BHC, LMXB \\
IGR J17597-2201 & $17^{\mathrm{h}} 59^{\mathrm{m}} 42^{\mathrm{s}}$ & $22^{\circ} 01^{\prime}$ & 1588 & $6.9 \pm 0.2$ & 179 & $2.8 \pm 0.4$ & NS, B, D, LMXB \\
IGR/SAX J18027-2017 & $18^{\mathrm{h}} 02^{\mathrm{m}} 42^{\mathrm{s}}$ & $20^{\circ} 17^{\prime}$ & 1274 & $4.4 \pm 0.2$ & 146 & $5.3 \pm 0.5$ & NS, P, HMXB? \\
\hline
\end{tabular}

- BHC - black hole candidate, NS - neutron star, HMXB - high mass X-ray binary, LMXB - low mass X-ray binary, B - burster, D- dipper, $\mathrm{P}$ - pulsar.

${ }^{a}$ Source coordinates as measured by INTEGRAL/IBIS.

${ }^{b}$ The effective exposure.

$c$ The average source flux in the energy band $18-60 \mathrm{keV}$, the flux was calculated assuming that the spectrum of the Crab nebula has the shape $\mathrm{d} N(E)=10.0 E^{-2.1} \mathrm{~d} E$.

${ }^{d}$ A maximum of the source flux in the $18-60 \mathrm{keV}$ energy band is given in parentheses.

e $3 \sigma$ upper limit.

The SPI spectrometer has a relatively poor angular resolution $\sim 2-2.5^{\circ}$ (Vedrenne et al. 2003) that precludes its use for point sources studies in such a crowded region as the Galactic Center. Therefore we do not use data from the SPI spectrometer.

The Galactic Center region was observed with the INTEGRAL observatory from August 23 till September 24, 2003 (Obs. IDs 0120213 and 0120016). The total exposure of these observations was slightly more than $2 \mathrm{Ms}$, but due to a decrease of the IBIS efficiency for off-axis sources their effective exposures were slightly lower (see Table 1). In order to investigate the variability of sources in hard X-rays on a time scale of half a year we used a set of observations of the Galactic Center field performed by INTEGRAL in March-April of 2004 with total exposure of $\sim 250 \mathrm{ks}$ (Obs. ID 0220133).

The data of all IBIS/ISGRI observations were processed with the method described by Revnivtsev et al. (2004a). In order to reconstruct the sources spectra from IBIS/ISGRI data we used a ratio of fluxes measured in different energy channels to the fluxes measured by the ISGRI detector from the Crab nebula in the same energy bands. Detailed analysis of Crab nebula observations suggests that with the approach and software employed, the conservative estimation of uncertainty in measurements of absolute fluxes from the sources is about $10 \%$ and the shape of the spectrum is about $5 \%$. The last value was added to the following spectral analysis as a systematic uncertainty in each energy channel.

The hard X-ray spectra were combined with spectra obtained with the PCA spectrometer $(3-20 \mathrm{keV})$ of the RXTE observatory (Bradt et al. 1993) where possible. In particular, we used publically available data of the PCA spectrometer of observations of IGR J17091-3624 (April 20, 2003, Obs. ID 80410-01-01-00) IGR J17464-3213 (from 26 Aug. through 23 Sep., Obs. ID 80146-01), IGR/XTE J17391-3021 (28 Aug., Obs. ID 80074-01), IGR/XTE J17597-2201 (June 3 and Oct. 11, 2001, Obs. ID 60117-02). The PCA spectrometer is sensitive to photons in the $3-20 \mathrm{keV}$ energy band, its effective area is $\sim 6400 \mathrm{~cm}^{2}$ at energies $6-7 \mathrm{keV}$, the energy resolution is $\sim 18 \%$ at these energies. For data reduction of the RXTE observations we used standard programs of the FTOOLS 5.3 package. In order to trace the long term variability of sources we have used the data of the all sky monitor (ASM) of RXTE in the 1.3-12.2 keV energy band (http://xte.mit.edu/ASM_lc.html).

\section{Results}

A list of sources with corresponding effective exposures and fluxes (in mCrab units) measured in the $18-60 \mathrm{keV}$ energy band is presented in Table 1 for 2003 and 2004. A flux of $1 \mathrm{mCrab}$ in this energy band corresponds to an energy flux of $\sim 1.4 \times 10^{-11} \mathrm{erg} \mathrm{s}^{-1} \mathrm{~cm}^{-2}$ for a source with a Crab-like spectrum with a photon index $\Gamma=2.1$. Only three sources were detected in the last series of observations; for the other two sources we obtained only upper limits. Most of sources have a steady behavior, except for two objects, IGR J17464-3213 and IGR/XTE J17391-3021, which demonstrated flares during observations. In Table 1 we present both average and maximum values of their fluxes. Model parameters of best-fit approximations of source spectra are listed in Table 2.

Below we will discuss each source in detail.

\section{IGR J17091-3624}

The source IGR J17091-3624 was discovered with the INTEGRAL observatory during Galactic Center observations in April, 2003 (Kuulkers et al. 2003). The source demonstrated hard X-ray emission up to $\sim 100 \mathrm{keV}$ with possible softening of the spectrum during subsequent INTEGRAL observations. Archival data of MIR/KVANT/TTM and BeppoSAX/WFC showed that this source was detected in soft X-rays from 1994 (Revnivtsev et al. 2003a; in't Zand et al. 2003). The region containing a new object was almost immediately observed with the RXTE observatory on April 20, 2003. Results of analysis of the RXTE data showed that the source has a flux of $\sim 4$ mCrab in the 3-20 keV energy band; its spectrum can be well approximated by a simple powerlaw model with a photon index of $\Gamma=1.43$ (Lutovinov \& Revnivtsev 2003). 
Table 2. Best-fit parameters of source spectra in the $>20 \mathrm{keV}$ energy band ${ }^{a}$.

\begin{tabular}{l|c|c|c}
\hline \hline \multirow{2}{*}{ Source } & \multicolumn{2}{|c|}{ Parameters } & \multirow{2}{*}{$\chi^{2}(N$ d.o.f. } \\
\cline { 2 - 3 } & Photon index & Energy, keV & \\
\hline IGR J17091-3624 & $2.23 \pm 0.06$ & $78^{b}$ & $6.8(10)$ \\
& $1.59 \pm 0.07^{c}$ & $52^{b}$ & $10.1(10)$ \\
IGR/XTE J17391-3021 & & $22 \pm 2^{d}$ & $2.7(6)$ \\
IGR J17464-3213 & $1.85 \pm 0.04$ & $136^{b}$ & $11.2(10)$ \\
IGR J17597-2201 & $1.70 \pm 0.07$ & $78 \pm 3^{e}$ & $37.0(32)^{f}$ \\
& $3.05 \pm 0.47^{c}$ & & $4.2(5)$ \\
IGR/SAX J18027-2017 & & $19 \pm 2^{d}$ & $1.1(5)$ \\
\hline
\end{tabular}

a Powerlaw, powerlaw with cutoff and bremsstrahlung models were used for the spectra approximations.

${ }^{b}$ Lower limits on the cutoff energy $(2 \sigma)$.

Best-fit parameters of sources spectra obtained in 2004; for IGR/SAX J18027-2017 they are same as in 2003.

$d$ The temperature of the bremsstrahlung emission.

$e$ The energy of the exponential cutoff.

$f$ The combined RXTE and INTEGRAL fit.

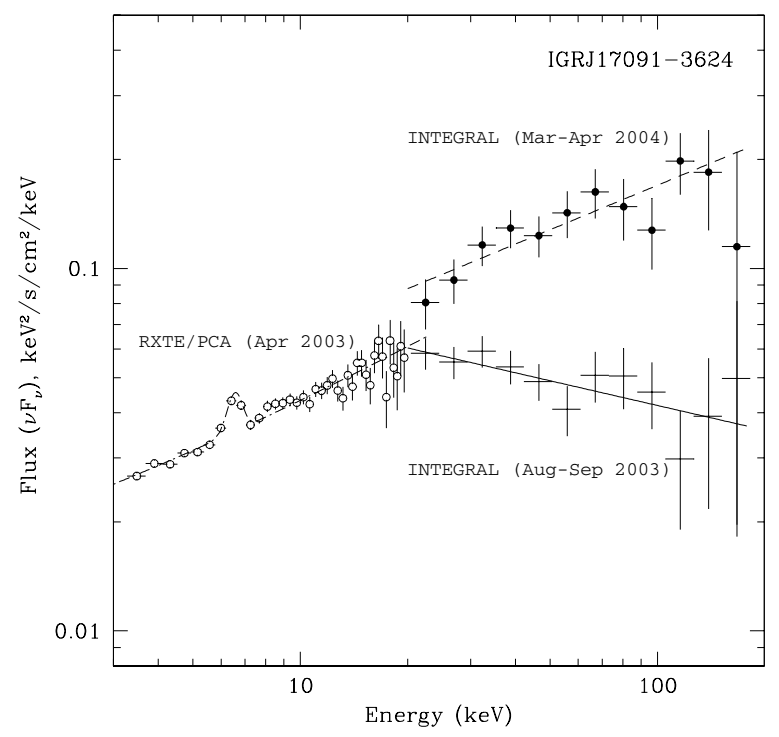

Fig. 1. The IGR J17091-3624 spectrum evolution in 2003-2004. Open circles represent RXTE data (Apr. 2003), crosses - INTEGRAL data (Aug.-Sep. 2003), dark circles - INTEGRAL data (Mar.-Apr. 2004). Corresponding best-fit approximations are shown by dash-dotted, solid and dashed lines, respectively.

A power-density spectrum of IGR J17091-3624 was described by the standard band-limited noise model typical for X-ray binary systems in the hard/low spectral state. Based on these results and taking into account the significant radio activity of IGR J17091-3624 (Rupen et al. 2003a), Lutovinov \& Revnivtsev (2003) assumed that this source is a possible blackhole candidate.

The source changed its brightness by a factor of $\sim 2$ between April 2003, Aug-Sep 2003 and April 2004 (see Table 1 and Kuulkers et al. 2003). Its spectrum in the energy band $20-150 \mathrm{keV}$ in 2003 can be described by a simple powerlaw with a photon index $\Gamma=2.23$ (Table 2, Fig. 1), that is a softer than it was in RXTE observations. As the source flux in the

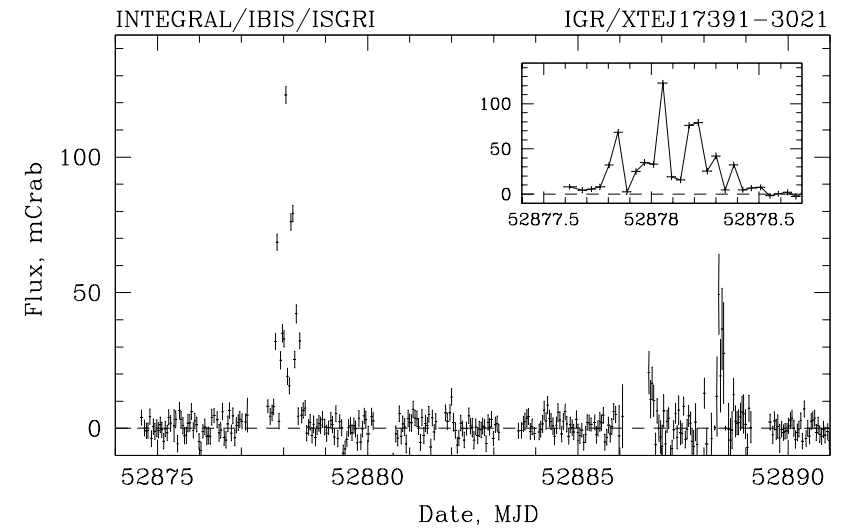

Fig. 2. INTEGRAL/IBIS/ISGRI light curve of IGR/XTE J17391-3021 in the $18-60 \mathrm{keV}$ energy band. Each point corresponds to a time bin of $\sim 3500 \mathrm{~s}$ (one pointing), error bars correspond to $1 \sigma$. A detailed profile of the strong outburst on 26-27 Aug., 2003 is shown in the inset.

hard X-ray energy band increased in 2004, its spectrum became harder; the photon index of the best fit power law approximation is $\Gamma=1.59$ (Fig. 1). Lower limits on the cutoff energy are $\sim 78$ and $\sim 52 \mathrm{keV}$, respectively (Table 2 ). The value of $\Gamma$ obtained in the April 2004 observations is close to those obtained with the RXTE observatory in 2003. The evolution of the spectrum of IGR J17091-3624 during approximately one year of observation is presented in Fig. 1. The spectrum of the source obtained by the RXTE observatory in 2003 was taken from Lutovinov \& Revnivtsev (2003).

\section{IGR/XTE J17391-3021}

On August 26, 2003 a strong outburst was detected by INTEGRAL during observations of the Galactic Center field (Sunyaev et al. 2003b). Coordinates of a possible new transient source IGR J17391-3021 were determined as RA = $17^{\mathrm{h}} 39^{\mathrm{m}} 06^{\mathrm{s}}$, Dec $=-30^{\circ} 21^{\prime} 30^{\prime \prime}$ with an accuracy of $3^{\prime}$. The following analysis showed that in spite of $\sim 5.5^{\prime}$ separation, the newly discovered source is likely to be the same transient source (XTE J1739-302) that was seen by the RXTE in 1998 (Smith et al. 1998). Therefore in the subsequent analysis and discussions we called this source the double name IGR/XTE J17391-3021.

Observations of this field performed with the Chandra observatory and VLA revealed inside the INTEGRAL coordinate error box two sources with non-overlapping coordinates: $\mathrm{RA}=17^{\mathrm{h}} 39^{\mathrm{m}} 11^{\mathrm{s}} .58$, Dec $=-30^{\circ} 20^{\prime} 37.6^{\prime \prime}$ (Chandra, an accuracy is better than a few arcsec, Smith \& Heindl 2003) and $\mathrm{RA}=17^{\mathrm{h}} 39^{\mathrm{m}} 01^{\mathrm{s}} .52$, Dec $=-30^{\circ} 19^{\prime} 34.9^{\prime \prime}($ VLA, an accuracy of $0.2^{\prime \prime}$, Rupen et al. 2003b). The distance between these two positions is quite large, indicating that the radio source may be not related to IGR/XTE J17391-3021.

The light curve of the source IGR/XTE J17391-3021 obtained with the INTEGRAL observatory in August-September, 2003 is presented in Fig. 2. The total length of the outburst was less than one day; its structure was complex and included several peaks. The maximal flux detected in the $18-60 \mathrm{keV}$ energy 


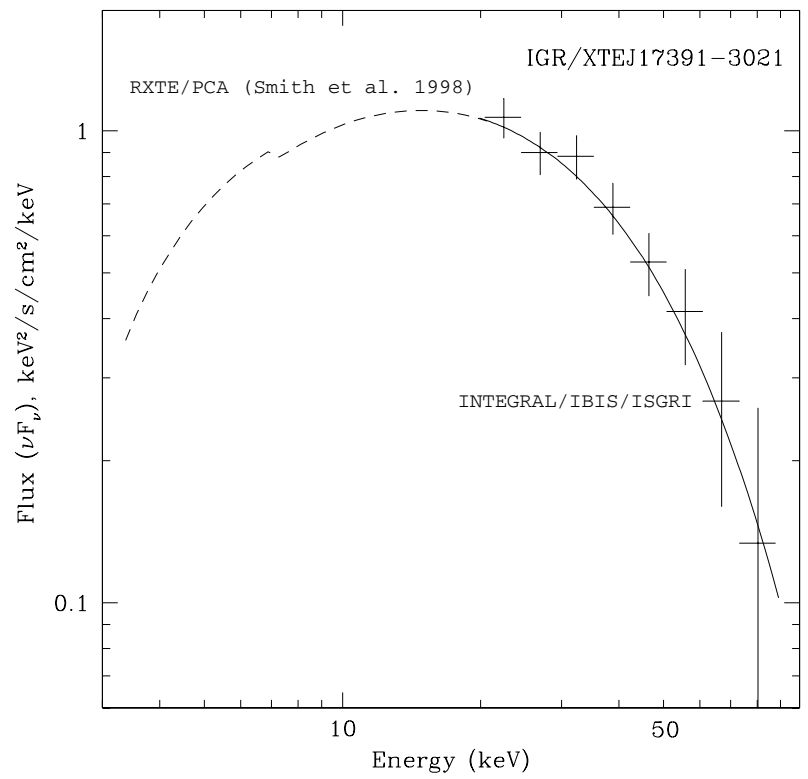

Fig. 3. The spectrum of IGR/XTE J17391-3021, measured with INTEGRAL (crosses) and its best-fit approximation (solid line). The best-fit approximation model of the source spectrum obtained with RXTE in 1998 is shown by a dashed line. We fixed its parameters to values obtained by Smith et al. (1998) and fitted only its normalization to the flux measured with INTEGRAL.

band is $\sim 120$ mCrab. The follow-up observations performed with the RXTE observatory on Aug. 28 gave us only an upper limit to the source flux of $\sim 3 \mathrm{mCrab}$ in the $3-20 \mathrm{keV}$ energy band. Thus the radio flux observed on this day by VLA in the INTEGRAL error circle (Rupen et al. 2003b) may be the reflection of the previous X-ray activity of the source or may be not related to IGR/XTE J17391-3021.

We reconstructed the spectrum of the source during its different flux values and found that the shape of the spectrum was practically identical. In the following analysis we used the energy spectrum of the source in the maximum of the outburst (Fig. 3). Approximation of this spectrum by a single power law (with $\Gamma \sim 3$ ) gives an unacceptably high $\chi^{2}$ value (10.8 for 5 d.o.f.). An optically thin thermal bremsstrahlung model or power law model with exponential cutoff provides much better fits (see Table 2). Such a spectral shape in hard X-rays is typical for of binary systems with neutron stars. The fit temperature was found to be the same as measured by Smith et al. (1998). These authors also reported that timing analyses of the source performed with the RXTE observatory have not show pulsations with an amplitude above $2 \%$ with a period less than $300 \mathrm{~s}$. Also no X-ray burst-like events were observed from the source. Thus the nature of IGR/XTE J17391-3021 is still unclear; however the observed spectral shape indicates that it is a neutron star binary, and very likely a high mass X-ray binary.

During INTEGRAL observations one more outburst with a complex structure was detected from IGR/XTE J17391-3021 (see Fig. 2). The maximum flux was $\sim 2$ times lower than that observed on 26-27 Aug.; the shape of the spectrum was the same.

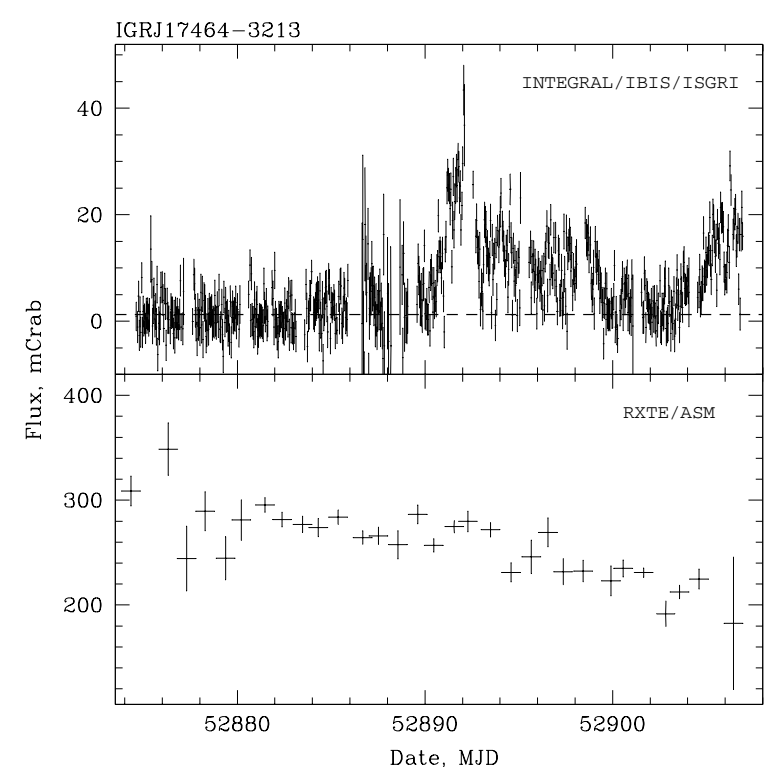

Fig. 4. Light curves of IGR J17464-3213 obtained in AugustSeptember 2003 with INTEGRAL/IBIS in the 18-60 keV energy band (upper panel) and RXTE/ASM in the 1.3-12.2 keV energy band (bottom panel). Each point in INTEGRAL data corresponds to a time bin of $\sim 3500 \mathrm{~s}$, in RXTE data - to the one-day averaging. Error bars corresponds to $1 \sigma$.

IGR J17464-3213 (=XTE J17464-3213 = H 1743-322)

The transient source IGR J17464-3213 was detected by the INTEGRAL observatory on March 21, 2003 (Revnivtsev et al. 2003b). During the next several days the source flux increased by a factor of $\sim 3$ and reached the value of $\sim 60-70 \mathrm{mCrab}$ in a wide energy band of 15-200 keV. Subsequent investigations (Markwardt \& Swank 2003a) showed that the position of the source is consistent with one of two equally possible positions of the source H1743-322, which was detected in 1977-1978 with HEAO-1 (Gursky et al. 1978). The follow-up observations with VLA revealed a probable radio counterpart of IGR J17464-3213 from which a strong radio flare was detected on April 8, 2003 (Rupen et al. 2003c). The Magellan optical observations revealed the possible counterpart in $R$ and $I$ filters (Steeghs et al. 2003). It was proposed that the source IGR J17464-3213 observed by INTEGRAL is a recurrent appearance of the source H1743322 (Parmar et al. 2003). The outburst lasted more than 200 days. Special TOO observations were performed with the INTEGRAL observatory during the rising part of the outburst and near its maximum (Parmar et al. 2003). These observations showed that the source demonstrated a behavior typical of $\mathrm{X}$-ray novae. Its spectrum in a wide energy band of 4-200 keV was well approximated by a simple powerlaw model with a photon index of $\sim 2.7$ with indications of a soft component, that is typical for the high/soft state of X-ray novae. The source monitoring performed with RXTE in May-July, 2004 allowed to observe it in several black hole states and revealed various types of variability, including QPOs (Homan et al. 2004).

At the moment of our observations in August-September 2003 the source flux in the RXTE/ASM energy band 


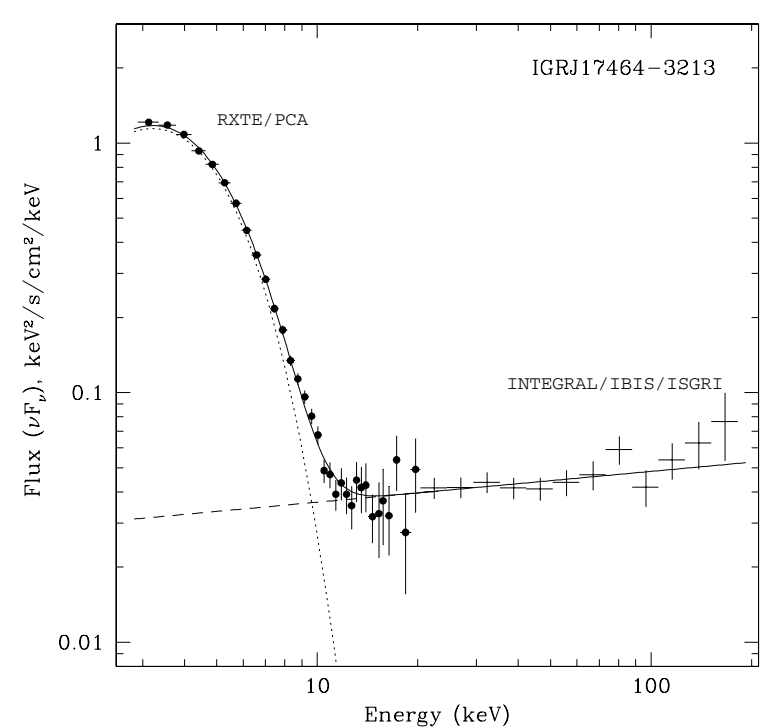

Fig. 5. Broadband spectrum of IGR J17464-3213 obtained with INTEGRAL (crosses) and RXTE (dark circles) observatories. Bestfit approximation with the blackbody+powerlaw model is presented by solid line, contributions of each component are shown by dotted and dashed lines, respectively.

(1.3-12.2 keV) dropped more than 2 times its maximal value and was still decreasing (see also Homan et al. 2004). In the hard energy band (18-60 keV) the source flux was practically constant at the level of $\sim 1.5$ mCrab during the first part of the observations and demonstrated outburst-like events with a maximum of $\sim 40 \mathrm{mCrab}$ during the second one (Fig. 4, Grebenev et al. 2003b). The spectra of the source in the hard $\mathrm{X}$-ray energy band did not change during these flux variations. In order to construct a broadband X-ray spectrum of the source we combined data of INTEGRAL and RXTE observatories (Fig. 5). The spectral points obtained with the RXTE/PCA were rescaled to match those of INTEGRAL/IBIS. The bestfit model of the broadband spectrum consists of two components: 1) a soft component which can be described by the blackbody disk emission (Shakura \& Sunyaev 1973) with the temperature $k T_{\text {in }}=1.05 \pm 0.01 \mathrm{keV} ; 2$ ) a hard powerlaw tail with $\Gamma=1.85 \pm 0.04$. This tail was visible up to $\sim 200 \mathrm{keV}$ without any cutoff ( $2 \sigma$ lower limit on the cutoff energy is $\sim 136 \mathrm{keV}$, see Table 2).

Note that for our 2004 observations (April 2004) the outburst from IGR J17464-3213 was finished and we obtained only an upper limit on its flux (Table 1).

Summarizing all the above we can conclude that IGR J17464-3213 is a classical X-ray nova - a low mass X-ray binary harboring a black hole - which experienced a recurrent outburst in 2003.

\section{IGR J17597-2201}

The source IGR J17597-2201 was detected by INTEGRAL on March 30-April 1, 2003 with a flux of $\sim 5 \mathrm{mCrab}$ in the 15-40 keV energy band (Lutovinov et al. 2003). Two weeks later the source intensity increased to $10-15 \mathrm{mCrab}$ in the same energy band. Just after the discovery of this new transient

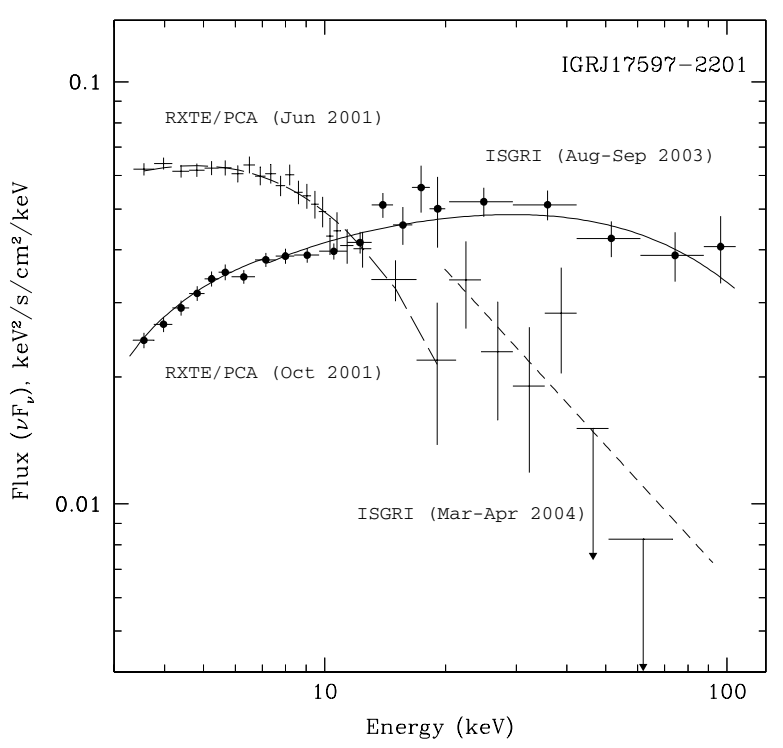

Fig. 6. IGR J17597-2201 spectrum changes observed by RXTE and INTEGRAL observatories in 2001 and 2003-2004, respectively. Crosses represent the high/soft state of the source, dark circles low/hard state. Corresponding best-fit approximations are shown by dashed and solid lines, respectively. The contribution of the galactic ridge to RXTE/PCA spectra was subtracted.

source, Markwardt \& Swank (2003b) reported that its position is consistent with a transient XTE J1759-220 which had been observed with RXTE since Feb., 2001, but this information was not published. The regular galactic bulge scans performed with RXTE showed that the source demonstrates a strong erratic flux variability in the standard X-ray band. There was an observational evidence of dipping behavior that implies high inclination of the binary. Also there were observed X-ray bursts, suggesting that the source is a neutron star binary. No QPO or pulsations were detected (Markwardt \& Swank 2003b).

IGR J17597-2201 was observed continuously during both series of our Galactic Center field observations. The source flux was 2.5 times higher in 2003 than in 2004 (see Table 1). Such a change in the source flux was accompanied by a strong change of its spectral shape (Fig. 6). During 2003 observations the source was hard $(\Gamma \sim 2.2)$ and its flux was detected up to $\sim 100 \mathrm{keV}$; during 2004 the source spectrum was much softer (see Fig. 6). Such a difference can be interpreted as a spectral transition from the hard/low to the soft/high state of the neutron star binary. As such a transition typically occurs in the luminosity range around $\sim 0.01 L_{\text {Edd }} \sim 10^{36} \mathrm{erg} \mathrm{s}^{-1}$ (see e.g. Maccarone 2003, and references therein), we can roughly (within a factor of $\sim 2$ ) estimate the distance to the source $d \sim 5-10 \mathrm{kpc}$, that may confirm that IGR J17597-2201 is located in the Galactic bulge.

In order to obtain a broadband energy spectrum of the source we used data from the RXTE archive (observations performed on June 3, 2001 and Oct. 11, 2001). For the reconstruction of the IGR J17597-2201 spectrum we should carefully take into account the contribution of the Galactic ridge emission to the flux detected by the RXTE/PCA (similar to what was done in Revnivtsev 2003a). To do this we predicted the spectrum of the Galactic ridge at the position of IGR J17597-2201 using the 
following assumptions. We have taken the spectral shape of the Galactic ridge emission in the form described by Revnivtsev (2003b). We assumed that most of the line blend emission ( $\sim 6-7 \mathrm{keV})$ detected by the RXTE/PCA at the position of IGR J17597-2201 comes from the Galactic ridge component. This assumption agrees well with parameters of the ridge emission presented in Valinia \& Marshall (1999) or Revnivtsev (2003b) and allow us to relatively accurately predict the flux of the Galactic ridge at the position of the source. The predicted Galactic ridge component was subtracted from the spectra observed by RXTE/PCA. Resulting broadband spectra are presented in Fig. 6.

The combined (INTEGRAL and RXTE) spectrum of IGR J17597-2201 in the hard state can be well described by a power law with an exponential cutoff at high energies - a model typical of neutron star binaries in the low/hard spectral state (see Table 2). The soft/high state spectrum of the source can be well approximated (due to limited statistics) by a thermal bremsstrahlung model with the temperature $k T=$ $7.5 \pm 0.5 \mathrm{keV}$. Such a spectral shape resembles those of neutron star binaries in their soft/high spectral state and the temperature parameter $k T$ strongly exceeds those of black holes binaries in their soft/high spectral state (cf. IGR J17464-3213 in Fig. 5). This conclusion support the suggestion of Markwardt \& Swank (2003b) about the nature of IGR J17597-2201.

\section{IGR/SAX J18027-2017}

First information about a detection of a possible new source IGR J18027-2017 near the bright X-ray source GX9+1 with the INTEGRAL observatory was discussed in April 2003 during INTEGRAL Core Program observations of the Galactic Center field. An analysis of the archival data of the BeppoSAX observatory revealed a source in the Galactic Center, which was named SAX J1802.7-2017 (Augello et al. 2003). The detailed timing analysis produced by Augello et al. (2003) showed that this source is an X-ray pulsar with a pulse period of $\sim 139.6 \mathrm{~s}$ and allowed one to determine binary parameters of the system.

IGR/SAX J18027-2017 was within the IBIS field of view during all the Galactic Center observations in 2003 and 2004. The flux registered from the source in the 18-60 keV energy band was nearly constant and equal in both sets of observations (see Table 1). The shape of the source spectrum in hard $\mathrm{X}$-rays did not change during observations. It can be described by a power law model with an exponential cutoff at high energies. The data of the IBIS telescope very poorly constrain the photon index of the powerlaw, but we can determine the energy of the cutoff $E_{\text {cut }} \sim 18 \mathrm{keV}$, that is typical for X-ray pulsars. In order to present a broadband spectrum of the source (Fig. 7), we have fitted the spectrum observed by IBIS/ISGRI with a typical pulsar spectral model (power law with exponential cutoff at high energies) while fixing the power law index on the value obtained by Augello et al. (2003) in the soft energy band. The best-fit value of the cutoff energy of $\sim 10 \mathrm{keV}$ resulting from such a procedure is lower than that obtained by fitting only hard X-ray data.

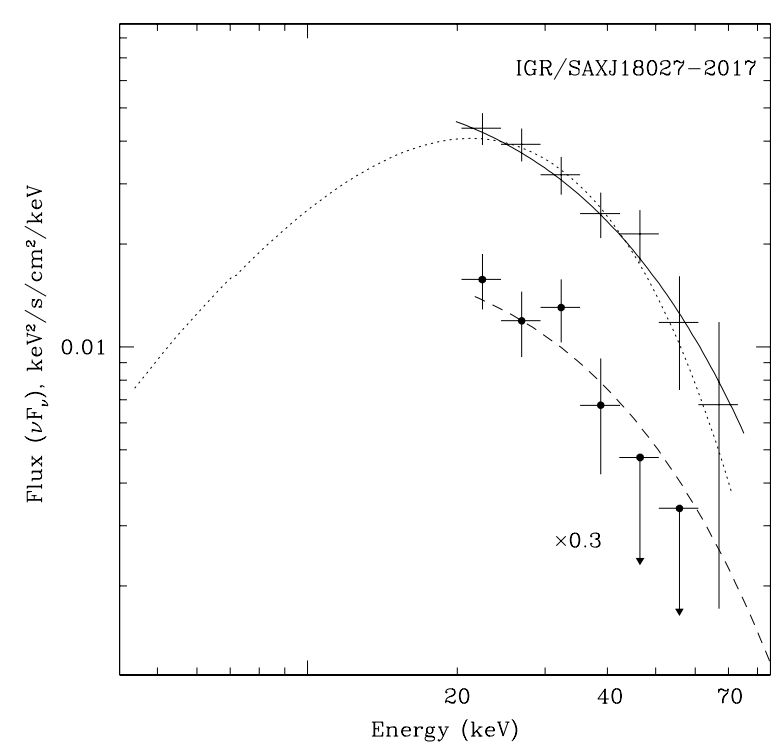

Fig. 7. Spectra of IGR/SAX J18027-2017 measured with INTEGRAL in 2003 (crosses) and 2004 (filled circles, scaled by a factor of 0.3). Solid and dashed lines represent corresponding best-fit approximations. An extrapolation of the spectral model obtained by BeppoSAX to a wide energy band is shown by dotted line (see text for detail).

\section{Summary}

We presented an analysis of INTEGRAL observations of five newly discovered sources - IGR J17091-3624, IGR/XTE J17391-3021, IGR J17464-3212, IGR J17597-2201 and SAX/IGR J18027-2017. Taking into account the mentioned above information about the sources we can conclude that all of them have a Galactic origin.

The nature of these sources can be summarized as following:

- IGR J17091-3624 is a likely black hole candidate.

- Properties of IGR/XTE J17391-3021 indicate that it is likely a high-mass neutron star binary. It is possible that it can be an accreting X-ray pulsar.

- IGR J17464-3212 is a classical X-ray nova harboring a black hole, likely a low mass X-ray binary.

- IGR J17597-2201 is a low mass X-ray binary, harboring a neutron star.

- SAX/IGR J18027-2017 is a neutron star binary, accreting $\mathrm{X}$-ray pulsar, likely HMXB.

Acknowledgements. The authors thank Eugene Churazov for developing of methods of analysis of the IBIS data and software. We would like to thank Marat Gilfanov and referee, Arvind Parmar, for useful comments. This research has made use of data obtained through the INTEGRAL Science Data Center (ISDC), Versoix, Switzerland, Russian INTEGRAL Science Data Center (RSDC), Moscow, Russia, and High Energy Astrophysics Science Archive Research Center Online Service, provided by the NASA/Goddard Space Flight Center. This work was partially supported by grants of Minpromnauka NSH-2083.2003.2 and 40.022.1.1.1102 and the program of Russian Academy of Sciences "Non-stationary phenomena in astronomy". A.L., M.R. and S.M. acknowledge the support of RFFI grant 04-02-17276 and the International Space Science Institute (ISSI, Bern). 


\section{References}

Augello, G., Iaria, R., Robba, N., et al. 2003, ApJ, 596, L63 Bird, T., Barlow, E., Bassani, L., et al. 2004, ApJ, 607, L33

Bradt, H., Rothschild, R., \& Swank, J. 1993, A\&AS, 97, 355

Churazov, E., Gilfanov, M., Sunyaev, R., et al. 1994, ApJS, 92, 381

Eismont, N., Ditrikh, A., Janin, G., et al. 2003, A\&A, 411, L37

Gonzalez-Riestra, R., Oosterbroek, T., Kuulkers, E., Orr, A., \& Parmar, A. N. 2004, A\&A, 420, 589

Grebenev, S., Sunyaev, R., Lutovinov, A., et al. 2003a, Astron. Telegram, 192, 1

Grebenev, S., Sunyaev, R., \& Lutovinov, A. 2003b, Astron. Telegram, 189,1

Gursky, H., Bradt, H., Doxsey, R., et al. 1978, ApJ, 223, 973

Homan, J., Miller, J., Wijnands, R., et al. 2004 [arXiv:astro-ph/0406334)]

Kuulkers, E., Lutovinov, A., Parmar, A., et al. 2003, Astron. Telegram, 149,1

in't Zand, J., Heise, J., Lowes, P., et al., Astron. Telegram, 160, 1

in't Zand, J., Heise, J., Ubertini, P., et al., Proc. V INTEGRAL Workshop, in press [arXiv:astro-ph/0407085]

Lebrun, F., Leray, J. P., Lavocat, P., et al. 2003, A\&A, 411, L141

Lutovinov, A., \& Revnivtsev, M. 2003, Astron. Lett., 29, 719

Lutovinov, A., Walter, R., Belanger, G., et al. 2003, Astron. Telegram, 155,1

Maccarone, T. 2003, A\&A, 409, 697

Markwardt, C., \& Swank, J. 2003a, Astron. Telegram, 136, 1

Markwardt, C., \& Swank, J. 2003b, Astron. Telegram, 156, 1

Molkov, S., Cherepashchuk, A., Lutovinov, A., et al. 2004, Astr. Lett., 30,534

Parmar, A., Kuulkers, E., Oostrebroek, T., et al. 2003, Astr. Lett., 411, L421

Revnivtsev, M. 2003a, Astr. Lett., 29, 644

Revnivtsev, M. 2003b, A\&A, 410, 865
Revnivtsev, M., Gilfanov, M., Churazov, E., \& Sunyaev, R. 2003a, Astron. Telegram, 150, 1

Revnivtsev, M., Chernyakova, M., Capitanio, F., et al. 2003b, Astron. Telegram, 132, 1

Revnivtsev, M., Sunyaev, R., Varshalovich, D., et al. 2004a, Astr. Lett., 30, 382

Revnivtsev, M., Sunyaev, R., Gilfanov, M., et al. 2004b, Astr. Lett., 30,582

Rupen, M., Mioduszewski, A., \& Dhawan, V. 2003a, Astron. Telegram, 152, 1

Rupen, M., Mioduszewski, A., \& Dhawan, V. 2003b, Astron. Telegram, 184, 1

Rupen, M., Mioduszewski, A., \& Dhawan, V. 2003c, Astron. Telegram, 142, 1

Sakano, M., Koyama, K., Murakami, H., Maeda, Y., \& Yamauchi, S. 2002, ApJS, 138, 19

Shakura, N., \& Sunyaev, R. 1973, A\&A, 24, 337

Smith, D., Main, D., Marchall, F., et al. 1998, ApJ, 501, L181

Smith, D., \& Heindl, W. 2003, Astron. Telegram, 218, 1

Steeghs, D., Miller, J., Kaplan, D., \& Rupen, M. 2003, Astron. Telegram, 146, 1

Sunyaev, R., Grebenev, S., Lutovinov, A., et al. 2003a, Astron. Telegram, 190, 1

Sunyaev, R., Lutovinov, A., Molkov, S., \& Deluit, S. 2003b, Astron. Telegram, 181, 1

Tomsick, J., Lingenfelter, R., Corbel, C., et al. 2004, Proc. V INTEGRAL Workshop, in press [arXiv: astro-ph/0404420]

Ubertini, P., Lebrun, F., Di Cocco, G., et al. 2003, A\&A, 411, L131

Valinia, A., \& Marshall, F. 1998, ApJ, 505, 134

Vedrenne, G., Rogues, J.-P., Schönfelder, V., et al. 2003, A\&A, 411, L63

Winkler, C., Courvoisier, T., Di Cocco, G., et al. 2003, A\&A, 411, L1 